\section{Tear Film Breakup Time in Diabetic Patients}

Sir,

Keratoconjunctivitis sicca commonly called 'dry eye' has many causative factors; and diabetes is identified as one of the etiology of dry eye syndrome worldwide. Diabetes is one of the leading causes of ocular morbidity and blindness in 20-74 years of age. ${ }^{1}$ As it affect not only the anterior, posterior segment of the eye and lens, but also the retina leading to retinopathy. According to WHO, the prevalence of diabetes in Pakistan 2016 is $9.8 \%$. The reported prevalence of dry eye syndrome is $15-33 \%$ in those of over 65 years of age. ${ }^{2}$

Tear film has three layers: an oily (lipid) layer, a watery (aqueous) layer and a mucin layer. The main function of these three layers is to help maintain the health of our eyes and ward off infection. Tear breakup time (TBUT) is an objective test, which is done to diagnose dry eye disease. Fluorescein staining had the highest specificity $(97 \%) .^{3}$ A tear breakup time under 10 seconds is considered abnormal. Various former research trials haveestablished the association between diabetes and dry eye disease.

This study, which was a hospital based cross-sectional study, enrolled 120 patients from OPD of Holy Family Hospital from $1^{\text {st }}$ November till $31^{\text {st }}$ December 2017. Patients with diagnosed Diabetes were selected. All other causes of dry eye like autoimmune disease, vitamin A deficiency, medication e.g. antihistamine, antidepressants, hormonal therapy, laser surgery, postmenopausal females and smoking were also excluded. Tear breakup time (TBUT) test was then performed to assess for dry eye disease with value of less than 10 seconds indicated diseased. Sixty two out of $120(51.7 \%)$ patients had TBUT less than 10 seconds $(p=$ $0.04)$ with $56.5 \%(n=34)$ males and $43.5 \%(n=26)$ females affected; however, the gender distribution was equivalent constituting 60 each in sample. In contrast,a study in Brazil showed female predominance $(75.9 \%)$ as opposed to this study. Sixteen (13.3\%) patients were aged between $20-40$ years, $64.2 \%(n=77)$ were from $41-60$ years, $21.7 \%(n=27)$ were from $61-80$ years, and $0.8 \%(n=1)$ greater than 80 years showing the peak frequency at 41-60 years of age. Patients with Type 1 diabetes had greater frequency $(70 \%)(n=84)$ to develop dry eye syndrome. Forty-three $(35.8 \%)$ patients were suffering from diabetes for $1-5$ years, $28.3 \%$ $(n=34)$ for $6-10$ years, and $35.8 \%(n=43)$ for more than 10 years showing that the disease had a bimodal distribution,appearing eitherin first five years or after 10 years of diabetes.

Hence, this study showed that diabetes is a causative agent of dry eye syndrome, but gender distribution is still debatable, for which more research work should be done to negotiate thisfact. None of the studies done previously, demonstrated the bimodal distribution of dry eye syndrome with duration of diabetes, which is unique in this study. Further clinical trials should be performed to clarify this fact. Moreover, research programmes should be carried out for symptoms of diabetes associated dry eye syndrome, its association with glycated hemoglobin $\mathrm{HgAlc}$, its correlation with diabetic retinopathy and effect on its incidence with prevention measures of diabetes. Limitations in diagnostic approach for dry eye syndrome in diabetes are mainly due to lack of objective tests with sufficient sensitivity and specificity that could beapplied easily in routine clinical care settings; and at the same time be adequately reproducible. ${ }^{4,5}$ The therapeutic strategies of dry eye syndrome include artificial tears and lubrication, strict diabetic control, and behavioural modifications.

\section{CONFLICT OF INTEREST:}

No conflictofinterest is present in thisstudy.

\section{AUTHORS' CONTRIBUTION:}

SZ: Conception, design, data acquisition and analysis, drafting and critical revision.

ES: Data analysis and interpretation, critical revision and final approval.

\section{REFERENCES}

1. Harrison TR. Diabetes mellitus, In: Branwald E, Fauci S, Kasper D, Hauser LS, L Longo DJameson JL, editor. Harrison Principle of Internal Medicine 15 USA, MC Grow-Hil/2001; 2121.

2. Dry eye syndrome, NICE CKS, September 2012.

3. Najafi L, Malek M, Valojerdi AE, Khamseh ME, Aghaei H. Dry eye disease in type 2 diabetes mellitus; comparison of the tear osmolarity test with other common diagnostic tests: A diagnostic accuracy study using STARD standard.J Diabetes MetabDisord 2015; 14:39.

4. Bron AJ, Smith JA, Calonge M. Methodologies to diagnose and monitor dry eye disease. In: 2007 report of the international dry eye workshop (DEWS). Diagnostic methodology subcommittee of the international dry eye workshop. Ocul Surf 2007;5(2):108-23.

5. Lemp MA, Bron AJ, Baudouin CH, Benítez Del Castillo JM, Geffen D. Tear osmolarity in the diagnosis and management of dry eye disease. Am J Ophthalmol 2011; 151(5):792-8.

Saliha Zaman and Eileen Samuel

Shaukat Khanum Memorial Cancer Hospital, Lahore, Pakistan

Correspondence to: Dr. Eileen Samuel, Department of Radiation Oncology, Shaukat Khanum Memorial Cancer Hospital, Lahore, Pakistan

E-mail: eileensamuel@ymail.com

Received: November 30, 2018; Revised: September 03, 2019; Accepted: October 17, 2019

DOI: https://doi.org/10.29271/jcpsp.2020.07.774 